\title{
NSLS IN-VACUUM UNDULATORS AND MINI-BETA STRAIGHTS *
}

\author{
G. Rakowsky ${ }^{\dagger}$, D. Lynch, E.B. Blum and S. Krinsky \\ BNL, Upton, NY 11973, USA
}

\section{Abstract}

We review the work carried out in the X13 R\&D Straight Section of the NSLS X-Ray Ring on small gap in-vacuum undulators (IVUNs). Then we discuss: (1) plans to replace the pure permanent magnet undulator in $\mathrm{X} 13$ by a hybrid design providing stronger magnetic fields, enhancing the tunability of the device; (2) plans to install hybrid IVUNs in the two RF straights of the X-Ray Ring, increasing the number of insertion devices in the $\mathrm{X}$ Ray Ring to eight; (3) the possibility of reducing the vertical beta function in the X13 straight from $0.33 \mathrm{~m}$ down to $0.16 \mathrm{~m}$. This reduction in beta function would allow us to decrease the usable undulator gap from $3 \mathrm{~mm}$ down to $2 \mathrm{~mm}$, further increasing the tuning range.

\section{INTRODUCTION}

The NSLS has been pursuing a continuous program to improve machine performance and reliability and to increase optical flux and source brightness. Lattice improvements to reduce horizontal-to-vertical coupling from $1 \%$ to $0.1 \%$, as well as decrease vertical despersion, resulted in a reduction of vertical emittance $\varepsilon_{\mathrm{y}}$ by a factor of 10 , to $0.1 \mathrm{~nm}-\mathrm{rad}$, with a corresponding increase of brightness [1]. Beam current was steadily increased from $250 \mathrm{~mA}$ to $400 \mathrm{~mA}$. Additional lattice improvements reduced horizontal emittance from 90 to $45 \mathrm{~nm}$-rad, doubling the brightness [2]. Recently the beam energy has been increased from 2.584 to $2.8 \mathrm{GeV}$. (For now, beam current is restricted to about $300 \mathrm{~mA}$ for thermal reasons.)

In parallel with machine improvements, the NSLS has pioneered in the development of in-vacuum small-gap xray undulators. A comprehensive review of this effort has been published by Stefan [3], so only the highlights are given here.

The motivation for small-gap insertion devices (ID's) is well-known: reducing the gap $\mathrm{G}$ allows a proportionate reduction in period $\lambda_{u}$ for a given peak field $B_{u}$. In an undulator, the fundamental radiated wavelength is given by

$$
\begin{gathered}
\lambda_{\mathrm{o}}=\left(\lambda_{\mathrm{u}} / 2 \gamma^{2}\right)\left(1+\mathrm{K}^{2} / 2\right), \\
\text { where } \mathrm{K}=0.934 \mathrm{~B}_{\mathrm{u}}[\mathrm{T}] \lambda_{\mathrm{u}}[\mathrm{cm}] \\
\text { and peak field } \mathrm{B}_{\mathrm{u}} \propto \exp \left[-\pi \mathrm{G} / \lambda_{\mathrm{u}}\right]
\end{gathered}
$$

A shorter period produces a proportionately shorter resonant optical wavelength $\lambda_{\mathrm{o}}$, so higher-energy X-rays

\footnotetext{
* Work supported by the US Department of Energy under Contract DEAC02-98CH10886

†'E-mail: rakowsky@bnl.gov
}

can be obtained. Thus, small-gap ID's can extend a $2^{\text {nd }}$ generation machine's undulator spectral range into the realm of hard X-ray energies heretofore available only at the high-energy light sources.

One penalty for a smaller $\lambda_{\mathrm{u}}$ is a lower maximum $\mathrm{K}$ and a reduced tuning range as $\mathrm{K}$ varies with gap, so a trade-off between optical wavelength, flux and tuning range is necessary.

A secondary benefit of small-gap, short-period ID's is that for a given number of periods the device can be proportionately shorter. Thus, very compact ID's can be built, while still producing high brightness and high flux. One result is that multiple devices can share the relatively short NSLS straight sections, as is commonly done in the long straights at the APS and ESRF. For example, for several years now, our small-gap ID's have been sharing the X13 straight section with an Elliptically Polarized Wiggler [4].

An even more interesting result is that these very compact devices can fit into places previously unavailable for ID's, such as in the two RF straights at X9 and X29, as will be described later.

\subsection{The PSGU}

The first small-gap ID tested in the X13 straight was the Prototype Small-Gap Undulator (PSGU) [5], a purepermanent magnet (PPM) device with a period of $16 \mathrm{~mm}$, 18 periods (compared to the X21 wiggler's 13.5 periods) and a $6 \mathrm{~mm}$ minimum magnet gap. The device had a variable-gap vacuum chamber to study the physics of small-gap operation. The magnet arrays were in air, on a separate variable-gap mechanism, so the chamber gap and the magnet gap could be adjusted independently. This device confirmed that the X-Ray Ring could operate with gaps as small as $3 \mathrm{~mm}$ with good lifetime and no instabilities.

\subsection{The IVUN}

The present small-gap device in X13 is the In-Vacuum Undulator (IVUN) [6], built in a collaboration between Spring-8 and NSLS. It has a conventional, 4 block/period, Halbach-type PPM array with $11 \mathrm{~mm}$ period and 30.5 periods. Without the intervening vacuum chamber, the magnet gap can be closed down to the same gap as the PSGU vacuum chamber. At $3.3 \mathrm{~mm}$ gap, IVUN produces a peak field of $0.68 \mathrm{~T}$, with a $\mathrm{K}$ of 0.7 . Due to its larger number of poles, IVUN is also a very bright source at its $4.6 \mathrm{keV}$ fundamental. However, the relatively low $\mathrm{K}$ gives IVUN a limited tuning range. 


\section{HYBRID IVUN DESIGN}

The next stage of our in-vacuum ID development addresses the low $\mathrm{K}$ and limited tunability of the present IVUN. Some of the loss of K value can be recovered by employing a "hybrid" Halbach structure with permanent magnets and vanadium permendur poles, which produce a higher field than a PPM design. We have also tailored the tuning range of the next device to the needs of the structural biology program proposed for the new undulator beamline at X29. For macromolecular crystallography the energy range of interest is approximately $7-15 \mathrm{keV}$. Although this energy is too high to be reached by the fundamental of any practical undulator in a $2.8 \mathrm{GeV}$ ring, it can be covered by higher harmonics of a small-gap IVUN.

The new hybrid, in-vacuum Mini-Gap Undulator (MGU), now under construction, will have a $12.5 \mathrm{~mm}$ period and effectively 27 periods. The peak field at 3.3 $\mathrm{mm}$ gap, computed by the 3D magnetostatics code Radia [7] is $1.0 \mathrm{~T}$. This gives a $\mathrm{K}$ of 1.17 , a fundamental energy of $3.5 \mathrm{keV}$, and a tuning range of about $50 \%$ (3.5 - 5.5 $\mathrm{keV})$. The corresponding tuning range of the $3^{\text {rd }}$ harmonic will be $11-15 \mathrm{keV}$. Due to finite emittance, there is also a significant $2^{\text {nd }}$ harmonic on-axis, which will cover broadly $5-10 \mathrm{keV}$. Figure 1 shows a plot of brightness vs. photon energy for the MGU at a gap of $3.3 \mathrm{~mm}$ $(\mathrm{K}=1.17)$, together with the envelope of the peaks of the principal harmonics. The envelope curves have been calculated up to $\mathrm{K}=1.9$ to indicate the potential tuning range for operation down to a gap of $2 \mathrm{~mm}$.

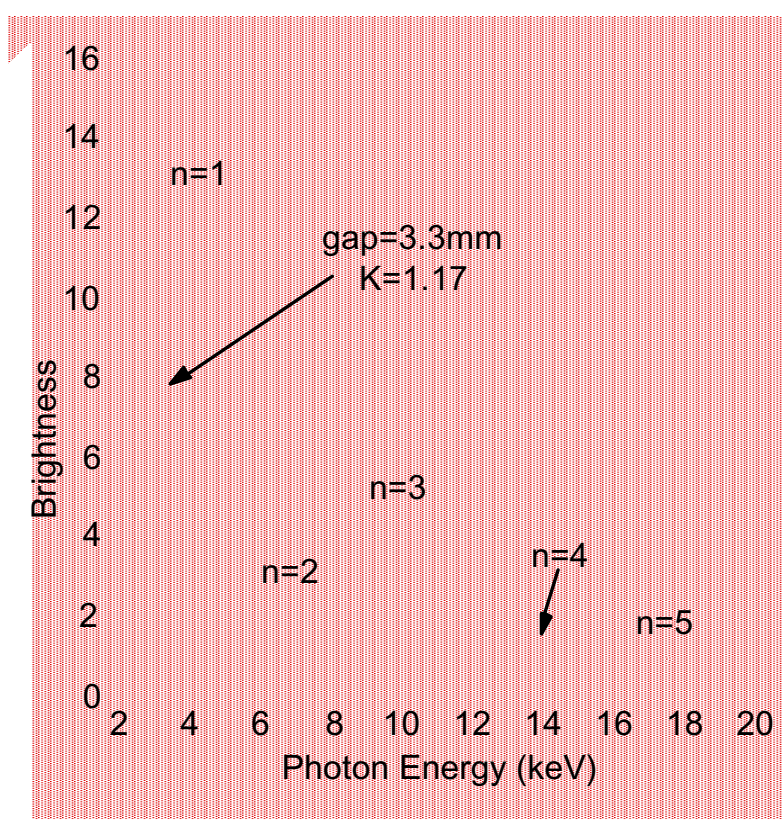

Figure 1. On-axis brightness of MGU $\left[\times 10^{17}\right.$ Photons $\left./ \mathrm{sec} / 0.1 \% \mathrm{bw} / 0.3 \mathrm{~A} / \mathrm{mm}^{2} / \mathrm{mr}^{2}\right]$. The spectrum for $\mathrm{K}=1.17$ (gap $=3.3 \mathrm{~mm})$ is overlaid with peak envelopes of the first 5 harmonics spanning $0.4<\mathrm{K}<1.9$.
Table 1 compares the key parameters of the PSGU, IVUN and MGU, scaled to the present energy of $2.8 \mathrm{GeV}$.

Table 1. Parameters of NSLS small-gap ID's

\begin{tabular}{|l|l|l|l|}
\hline & PSGU & IVUN & MGU \\
\hline Period $\lambda_{\mathrm{u}}$ & $16 \mathrm{~mm}$ & $11 \mathrm{~mm}$ & 12.5 \\
\hline Nom.Mag.Gap & $6.0 \mathrm{~mm}$ & $3.3 \mathrm{~mm}$ & $3.3 \mathrm{~mm}$ \\
\hline Peak Field $\mathrm{B}_{\mathrm{u}}$ & $0.62 \mathrm{~T}$ & $0.68 \mathrm{~T}$ & $1.0 \mathrm{~T}$ \\
\hline $\mathrm{K}_{\max }$ & 0.93 & 0.7 & 1.17 \\
\hline$E_{1}$ & $3.2 \mathrm{keV}$ & $5.4 \mathrm{keV}$ & $3.5 \mathrm{keV}$ \\
\hline e-beam Energy & $2.8 \mathrm{GeV}$ & $2.8 \mathrm{GeV}$ & $2.8 \mathrm{GeV}$ \\
\hline
\end{tabular}

The MGU magnetic structure is a conservatively designed, conventional Halbach "hybrid" with rectangular permendur poles and $\mathrm{NdFeB}$ magnets. A new, highenergy-product, high-temperature grade of $\mathrm{NdFeB}$ material (Sumitomo NEOMAX 39SH) will be used. It has $1.25 \mathrm{~T}$ remanence and a very high intrinsic coercivity $\left(\mathrm{H}_{\mathrm{ci}}>21 \mathrm{kOe}\right)$, to allow baking to $100^{\circ} \mathrm{C}$. Magnet coating is Ti-N for vacuum compatibility.

Due to the small dimensions, tight dimensional tolerances and precise pole positioning are required. Poles sit on the ground surface of an aluminum baseplate and are anchored by screws threaded into vented holes in the pole bottoms. Longitudinal indexing and pole alignment is accomplished by a tongue-and-groove interface between the pole ends and the aluminum side rails. Magnets fit into channels cut into the baseplate and into notches in the side rails and are held in pairs by clamps bearing on the magnets' chamfered corners. Some of these features can be seen in the photo of a 10-pole prototype of the MGU in Figure 2.

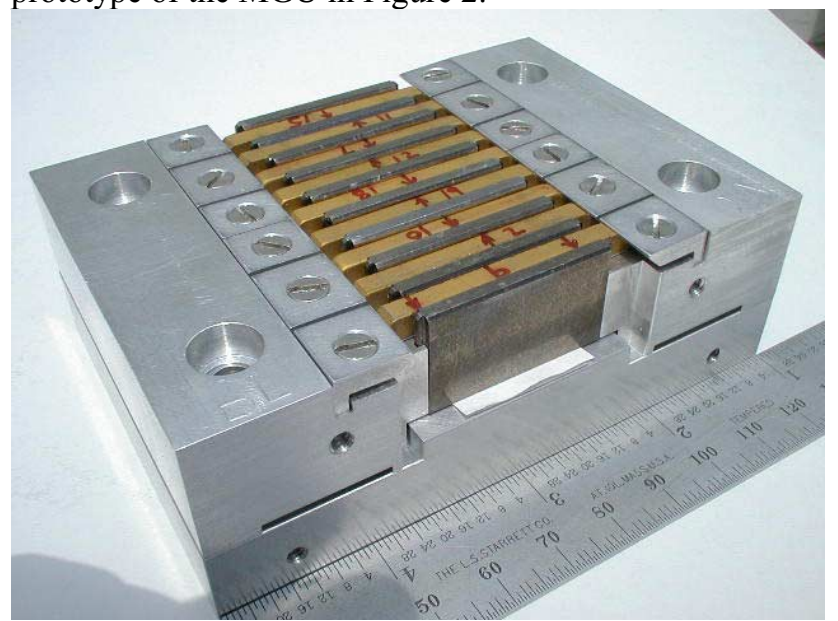

Figure 2. Ten-pole prototype of the MGU.

\subsection{End Termination Design}

An ideal ID termination is (a) non-steering and (b) displacement-free at each end, and (c) is gap-independent. Conditions (a) and (b) assure that the wiggle (radiation) axis is parallel to the beamline axis. These conditions are much harder to achieve in hybrid undulators than in PPM devices because of the nonlinear permeability of the poles. Halbach first suggested excitation patterns in electromagnetic wigglers that produce a binomial pattern of magnetic scalar potentials on the end poles to achieve 
non-steering (order 2) and/or displacement-free (order 3) terminations [8]. He also showed that if saturation is present in the poles, higher-order excitation patterns are needed for gap-independent termination. Recently hybrid undulators with higher-order terminations have been built at ESRF [9] and by STI [10], with varying volumes and/or strengths of the first three or four magnets at each end.

To design the MGU terminations, we modeled the undulator using Radia. For the MGU we have chosen a 4 th order termination, tapering the volumes of three end magnets and correcting a residual steering error by recessing the second pole. We will also attempt to maximize the number of useful poles by minimizing the field overshoot at the third pole.

A 10-pole prototype of the MGU has been built to verify the design. Initial Hall probe measurements agree with the calculated peak field. Some shimming was required to tune the termination. The resulting trajectories were fairly gap-independent. More detailed measurements are in progress.

\subsection{Mechanical Design}

Building on the success of the IVUN design, the MGU uses the same vacuum chamber and support structure, except for heavier through-vacuum members of the gap adjusting mechanism, to handle the higher magnetic forces in the MGU. The MGU will directly replace the IVUN in the X13 straight.

\section{NEW ID'S IN THE RF STRAIGHTS}

As part of the X-Ray Ring upgrade program, new, higher power RF cavities have been installed in the X29 straight. Their more compact design leaves a space between them in the center of the straight, which is just long enough to accommodate an MGU and a pair of isolation valves. Likewise, the planned replacement of the RF cavities in X9 will create a space for a future MGU. New vacuum chambers with a $0^{\circ}$ port are being built for the dipole magnets just downstream of X29 and X9 to serve the two new undulator beamlines.

At this writing, a second MGU, intended for the X29 straight, is in procurement and fabrication. Mechanically, it will share the same magnetic, vacuum and support structures as the X13 MGU, but with symmetric transitions and appropriate flanges to mate with isolation valves at the two RF cavities. A layout of an RF straight section with an MGU is shown in Figure 3.

\section{MINI-BETA LATTICE}

Lattice simulations show that it is possible to reduce $\beta_{y}$ locally at the center of a long X-Ray Ring straight section from $0.33 \mathrm{~m}$ to $0.16 \mathrm{~m}$ by adding additional quadrupoles just upstream and downstream of an MGU. This would further reduce the beam aperture, allowing MGU operation with gaps as small as $2 \mathrm{~mm}$. Reducing the undulator gap would increase the maximum $\mathrm{K}$ value and extend the tuning range to lower energies. The potential extended coverage is indicated in Figure 1.

There is no room in either the X13 or the RF straights for another pair of conventional quadrupoles. We are exploring miniaturized designs that would fit in the transition regions within the MGU envelope. Both the X13 and X29 MGU's will initially be installed without mini-beta quadrupoles.

\section{ACKNOWLEDGEMENT}

The authors thank W. Stoeber for mechanical design, S. Hulbert for spectral calculations and M. Lehecka and D. Harder for magnetic assembly and measurement.

\section{REFERENCES}

[1] J. Safranek and S. Krinsky, PAC'93, p.1491-1493.

[2].E.B. Blum et al., PAC'99, p.2304-2306.

[3] P.M. Stefan et al., NIM, A412 (1998) 161-173.

[4] E. Gluskin et al., PAC'95, p.1426-1428.

[5] P.M. Stefan et al., PAC'91, p.1096-1098.

[6] T. Tanabe et al., Rev. Sci. Instr.,69 (1), Jan. 1998.

[7] P. Elleaume et al., PAC'97, p.3509-3511.

[8] K. Halbach, NIM, A250 (1986) 95-99.

[9] J. Chavanne et al., PAC'97, p.3506-3508.

[10] S.C. Gottschalk et al., PAC'99, p.2674-2676.

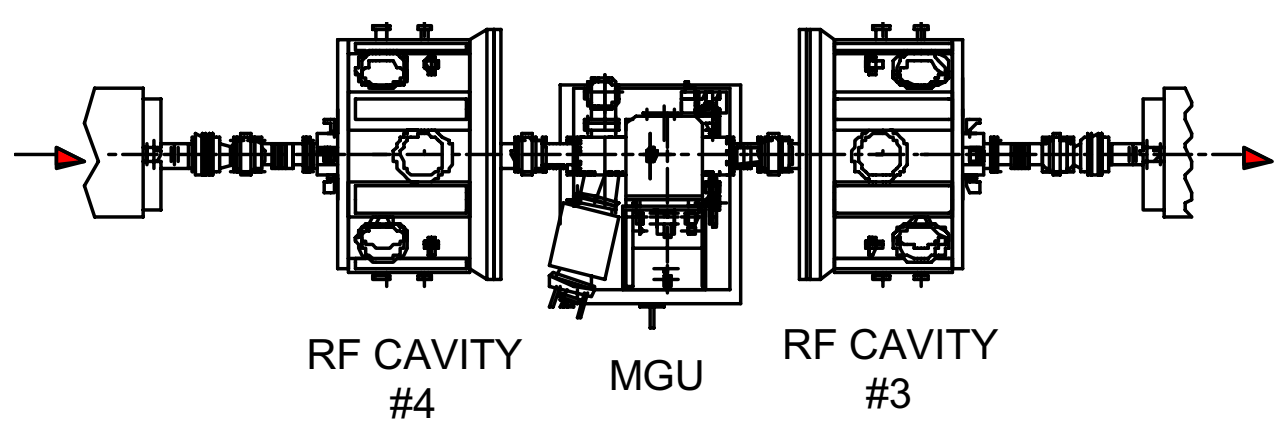

Figure 3. Layout of X-Ray Ring RF straight section with Mini-Gap Undulator in center. 